\title{
Physics Must Evolve Beyond the Physical
}

\author{
Deepak Chopra ${ }^{1}$ (D)
}

Received: 23 July 2018 / Accepted: 27 March 2019 / Published online: 29 April 2019

(C) The Author(s) 2019

\begin{abstract}
Contemporary physics finds itself pondering questions about mind and consciousness, an uncomfortable area for theorists. But historically, key figures at the founding of quantum theory assumed that reality was composed of two parts, mind and matter, which interacted with each other according to some new laws that they specified. This departure from the prior (classicalphysicalist) assumption that mind was a mere side effect of brain activity was such a startling proposal that it basically split physics in two, with one camp insisting that mind will ultimately be explained via physical processes in the brain and the other camp embracing mind as innate in creation and the key to understanding reality in its completeness. Henry Stapp made important contributions toward a coherent explanation by advocating John von Neumann's orthodox interpretation of quantum mechanics. von Neumann postulated that at its basis, quantum mechanics requires both a psychological and physical component. He was left, however, with a dualist view in which the psychological and physical aspects of QM remained unresolved. In this article, the relevant issues are laid out with the aim of finding a nondual explanation that allows mind and matter to exist as features of the same universal consciousness, in the hope that the critical insights of Planck, Heisenberg, Schrödinger, von Neumann, and Stapp will be recognized and valued, with the aim of an expanded physics that goes beyond physicalist dogma.
\end{abstract}

Keywords Stapp · Quantum reality $\cdot$ Linde $\cdot$ von Neumann $\cdot$ Orthodox interpretation $\cdot$ Consciousness

Modern physics speaks in the language of advanced mathematics, which makes it nearly impossible for an outsider to penetrate the discussion over vital issues at the heart of science. But mathematics in this case is not pure. It serves as an instrument for elucidating concepts about reality. If concepts are the primary stuff of science-I think we would all agree that this is true - then a certain nervousness begins to creep in. Concepts are a form of mental activity, and as soon as mind enters the picture, physics finds itself peering over the fence into the nonphysical.

The absolute need to transcend physical explanations brought me into contact with Henry Stapp a few years ago, and once we discovered that we shared a sense of urgency on the subject, we wrote a blog article, Is a Mind Element Needed to Interpret Quantum Mechanics? In the last century, theoretical physics has come to the realization that the world "out there" is disjointed from the mental world "in here." The

Deepak Chopra

carolyn@chopra.com

1 The Chopra Foundation, Carlsbad, CA, USA separation was reinforced by erecting a barrier between solid, concrete physical objects that can be explored objectively (through data collection, measurement, and experimentation) and the fickle, unstable, unreliable world of subjectivity.

Prof. Stapp has long argued that mind is essential for grasping not just quantum reality but reality as a whole. To summarize a few key points in our joint article, the founders of quantum theory assumed that reality was composed of two parts, mind and matter, which interacted with each other according to some new laws that they specified. This departure from the prior (classical-physicalist) assumption that mind was a mere side-effect of brain activity was such a startling proposal that it basically split physics in two, with one camp (the physicalists) insisting that matter alone, plus an element of quantum chance, determines every physical property of the universe, and the other camp embracing mind as the key to certain otherwise unexplained mysteries.

For several decades now, the first physicalist approach has been ascendant in the minds of many working physicists. But the founders of quantum mechanics were ambitious enough to want an explanation for the wholeness of reality. Some dominant figures, among them Max Planck, Erwin Schrödinger, and Werner Heisenberg, took a conceptual leap into the 
nonphysical, advocating that consciousness was an irreducible aspect of creation. Here's a sample of their public statements:

From Planck, speaking to a reporter from The Observer newspaper in 1931: "I regard consciousness as fundamental. I regard matter as derivative from consciousness. We cannot get behind consciousness. Everything that we talk about, everything that we regard as existing, postulates consciousness." From Schrödinger's 1944 book, What Is Life?: "Consciousness cannot be accounted for in physical terms. For consciousness is absolutely fundamental. It cannot be accounted for in terms of anything else." From Heisenberg, delivering a direct assault on conventional scientific thinking: "The atoms or elementary particles themselves are not real; they form a world of potentialities or possibilities rather than one of things or facts."

The intention behind these statements was scientific, indeed the ultimate in scientific thinking. As a mode of reductionism, physics in the early twentieth century had made a breakthrough into the substratum of the quantum. In their own minds, Planck, Schrödinger, and Heisenberg were going to an even more fundamental level of nature, following the implications of quantum behavior. If this was the intention, it did not bear fruit. The immediate effect, in fact, was to damage their reputations. They were accused of wandering into metaphysics, still a term of denigration in science. They had placed mind before matter, which if taken seriously would have undermined the whole scheme of "things" and "facts" that Heisenberg repudiated. Working physics remained firmly embedded in things and facts, and even though quantum theory backed up Heisenberg's depiction of pure potentiality (i.e., the wave function) and unpredictability at the core of nature, nobody saw their houses, cars, and labs vanishing into swirling quantum soup. With the classical world intact, business proceeded as usual.

So matters stood, with the mainstream of physics occupied with "things" and "facts," while on the fringes, the issue of mind was an exotic preoccupation of the few. But some mainstream theorists are grappling with the insight that consciousness cannot be left out of any valid explanation of nature as a whole. In a 1998 paper titled, with characteristically sweeping ambition, Universe, Life, Consciousness, the noted Russian-American physicist Andrei Linde rethought the relationship between physics and reality. "According to standard materialistic doctrine," Linde asserted, "consciousness ... plays a secondary, subservient role, being considered just a function of matter and a tool for the description of the truly existing material world."

But matter is not actually the starting point of science, Linde pointed out. The real starting point is our perceptions, which we take to be trustworthy. Any model of reality that regards the world "out there" as a given is mistaken. What we describe as the world "out there" is a substitute for perception, and therefore only theoretical, while perception itself, without a doubt, is real to us. In other words, Linde was telling his fellow scientists that they had reversed the order of things by believing that theory was more trustworthy than direct experience. "The [materialistic] theory is so successful that we almost never think about its limitations until we must address some really deep issues, which do not fit into our model of reality."

Those "really deep issues" skirt metaphysics, which remains anathema to physicists. One way to frame the discussion is through what Stapp and others have labeled the "mindlike" behavior of the physical world. In what way does the universe display mind-like behavior? To return to the points made in the article co-written with Stapp, the basic features of the evolving universe that bring the action of mind into play are choices.

For anything to actually "happen" in quantum mechanics, two things have to occur. First, a person has to ask a yes/no question about whether they will have a certain experience or not, then nature has to choose the answer to their question, yes or no. So, there are two choices being made, one is the choice of the question being asked, and the second is the choice of the answer being given.

The mathematical machinery of quantum mechanics is built around these paired choices of questions and answers. The answer to the posed question is specified by the famous quantum random choice. But how is the question chosen? The answer is "by the mind of an observing agent, on the basis of the perceived needs of that organism." Thus, the key choices that play an essential role in the evolution of the universe come from mind.

Freeman Dyson, who has been among the most eloquent and outspoken physicists on the matter, wrote in support of the same idea, "Matter in quantum mechanics is not an inert substance, but an active agent, constantly making choices between alternate possibilities-It appears that mind, as manifested by the capacity to make choices, is to some extent inherent in every electron."

Before the quantum revolution, billiard balls were often used as examples in physics because they neatly illustrate the laws of motion set down by Newton. Quantum mechanics replaced the table of billiard balls by a table filled with probability distributions of particles. But probability distributions of particles are not the same as real distributions of particles. They are in some sense imaginary. They can be distributions existing in the imaginations or minds of human beings. The same holds for anything composed of elementary particles - as Hans Peter Durr, the former director of the Max Planck Institute declared, "Matter is not made of matter." Rather, matter is a human construct, an interpretation of human perceptual activity. In any case, the notion of the probability distribution of particles seems to exist in a mind. But in quantum theory, the basic "material" aspects of nature are essentially just these probability distributions. Consequently, both the mind-like and matter-like aspects of nature seem to be essentially mind-like.

Quantum mechanics, as understood by its founders, shifted the foundations of physical science from what is described in 
physical terms (i.e., numbers attached to a space-time point) to our human experiences pertaining to such properties. The observer was no longer cut off from the observation. The Copenhagen interpretation of quantum mechanics $(\mathrm{QM})$ is in some sense dualistic, because it contained two parts: one part described in psychological/mental terms and another part described in physical/materialistic terms. In the quantum description of what is happening, these two parts are causally entwined: the observer participates in the effects upon the physical world that they are observing.

Whereas Newton's physical universe was an actual "thing," in QM, the physical aspect represents a mere "potentiality" for what can become actual. The universe does not behave like material stuff. There are abrupt jumps, leaps, and changes of state at the heart of quantum behavior. These sudden responses to increments in knowledge make the material aspect more mind-like than matter-like. Hence, both the "material" part and the "mental" part are mind-like in character.

I will not detail Stapp's advocacy of the orthodox interpretation of quantum mechanics devised by the brilliant HungarianAmerican theorist John von Neumann (for anyone interested, a complete explanation is contained in our article, which is easily found online). But the fact that the known laws for the physical state of the particles cannot explain how they behave is critical here. Something beyond the physical state is involved, asserts von Neumann and Stapp, echoing a remark made decades earlier by Heisenberg: "What we observe is not nature itself, but nature exposed to our method of questioning."

The notion of a participatory universe, associated with the famous Princeton physicist John Archibald Wheeler, holds great appeal. It accords with our subjective feelings. We do not feel like children with our noses pressed against the bakeshop window. We feel like characters woven into the fabric of life. Is this actually a viable opening for a new conception of reality? At the conceptual level, science is stuck with the demon of dualism, in that pure physicalism cannot explain mind, and mind seems too far removed from the physical universe to be its source.

The horizon of a new physics is just here, in overcoming duality, finding a unified explanation for matter and mind. Von Neumann, like his predecessors who took consciousness seriously, was left with dualism in the end, since the psychological and physical aspects of quantum mechanics interacted in some complex way but were not reducible to the same fundamental "stuff." In philosophy, the typical term for a unified conception of reality is monism. In modern cosmology, there is a trend toward monism that hybridizes mind and matter. Panpsychism, for example, which imparts consciousness or perhaps the seeds of consciousness, in all material things, is an attempt at a monistic explanation.

But these attempts are almost always motivated by an agenda, to maintain the primacy of the physical world. To say that an atom, a quark, a crystal, or a galaxy has consciousness actually explains nothing. One is simply restating the observation that the universe displays mind-like behavior. The essential issuer of choice, as Stapp states it, remains, only this time it can be writ large. The choice is between two explanations that are irreconcilable. Either mind created matter or vice versa. Science has lived for a long time with the assumption that the brain creates mind as a kind of byproduct the way a bonfire throws off heat. But this assumption has never been proven. The brain certainly displays physical activity that parallels thinking, feeling, and perceiving, but that proves nothing - a piano's keys and hammers move when Mozart is being played on it, but that does not mean the piano composed the music. In essence, neuroscience has adopted the same fallacious explanation. This does not hide the bald fact, however, that no physicalist has ever located the point at which common atoms of carbon, oxygen, nitrogen, and hydrogen learned to think.

The other choice, which faces anyone who wants to make consciousness the primary "stuff" of creation, is to show how mind makes matter appear. Surprisingly, this alternative is by far the stronger. When we have a thought, electrical and chemical impulses course through the brain and central nervous system. At the level of the synapse, the gap between two nerve endings, there is the creation of intermittent electrical charges and molecules of neurotransmitters. These are not created randomly; they express in physical coding a mental event. But beyond this obvious example of mind over matter, the very fact that we perceive the physical world "out there" is entirely dependent upon mind, and the means by which this transformation occurs remains a mystery.

The most basic natural phenomena do not exist outside our experience of them. For example, photons are not visible or bright. Their invisible status is altered only by contact with the retina at the back of the eye and the signals it sends along the optic nerve. On their journey to the visual cortex, these signals are also not visible, pictorial, or bright. The environment of the brain is dark, moist, and squishy. Physicalists can point out that a certain level of electromagnetic emission in neural tissue is analogous to light, but this does nothing to explain how one set of neural signals turns into the Grand Canyon and another the face of a friend, or anything else. There are no qualities of the physical world - through sight, sound, touch, taste, and smell- that are not mental artifacts.

A skeptic can argue that this transformation of raw neural signals into the appearance of the visible world is a brain process, not a mental one, but the argument does not hold water, because it traces back to the same dead end as all physicalist explanations: it is just as improbable that carbon, hydrogen, oxygen, and nitrogen atoms learned to see as to think.

But there is another fact that has not been addressed, and it goes to the heart of the matter. Reality cannot be modeled, which is to say that no matter how convincing any theory is, something will be left out. Physicalism for all intents and purposes leaves 
out mind. But the problem is far more universal. Models are only right about what they include and are inevitably wrong about what they exclude (I am simplifying but I hope not misrepresenting what Gödel's theorems proved logically).

To understand reality without a model, there is only one possibility: It must be experienced. This, at the bottom, is what everyone I have quoted has been saying. Just as there is no model for reality, there is no model for consciousness. If a super-hyperbrain scan in the future could be attached to a poet's head as he wrote a sonnet or a composer as he wrote a symphony, it could not replicate the creative process that converts daily brain activity into something beautiful, profound, and meaningful. Consciousness allows us to create concepts about the world, but consciousness itself is inconceivable.

Creativity is the emergence of something out of nothing. We marvel when the creative act produces Shakespeare's plays and Beethoven's symphonies, but "something out of nothing" is a critical issue in contemporary physics. What is causing the current unease among the "things" and "facts" mainstream scientists is quite astonishing. Empiricism has dwindled to the vanishing point. The pre-created state of the universe can only be conjectured mathematically, and as the late Stephen Hawking was at pains to point out in his last years, there is a discrepancy between theory and reality in the absence of empirical evidence.

We cannot conceive in non-mathematical thought of what came before time because the question itself is invalid - "before" only has meaning after time appeared on the scene. Likewise, we cannot conceptualize, outside advanced mathematics, what lies outside space - "outside" has meaning only after space appears. Since the human brain evolved through the workings of space, time, matter, and energy, the limits of mental concepts are real.

To experience reality without conceptual models is not a futile project; however, I would offer the possibility that there is a natural science of consciousness. In this science, what we now label the universe can be re-imagined as a mode of consciousness, seamlessly woven into the other modes that consciousness takes, such as reason, emotion, perception, interpretation, behavior, the working of nature across the span of evolution, and so on. That these modes of consciousness are viewed from a specific locality - the human viewpoint - means that in a very real sense, we live in a human universe.

This is not a choice. It is forced upon us by the fact that we are a species of consciousness different and unique, pursuing our own evolutionary track. Cave paintings emerged, for example, around 40,000 years ago, predating the accepted date for the appearance of the modern human brain around 20,000 years ago. The first artifacts that can be called humanoid sculptures date back between 200,000 and 700,000 years ago. Primitive toolmaking may extend as far back as our hominid ancestors more than a million years ago. We can dig up and collect visible evidence, but what is really at work here, what is evolving over time, is the unfoldment of the species of consciousness we identify with as Homo sapiens.
Once evolution is founded upon the ground state of consciousness, there is a level playing field between "mind" and "mind-like." It is no longer necessary to argue by analogy. The existence of mind is irrefutable and rests on far more solid ground than particles, which are human conceptions constructed from the capacities of the human brain with all its limitations. If I had to pick one word for the process that causes creation to appear, it would be reification. To reify is to take an idea and make it physical. We do it all the time. The Wright brothers' first airplane reified the idea of flying. If I jump at the sound of a car backfiring, my jolt of fear is reified into cortisol coursing through my bloodstream.

Reification closes the circle drawn by the quantum pioneers who dematerialized the physical universe. Now we can see how to bring it back again. Consciousness reifies whatever it chooses to make flesh. The creative act is unbounded, because consciousness is the observer and observed, the seer and the seen, and the experiencer and the experienced. Since science and technology cannot be stopped, reification is shaping our future. We would do a much better job, however, if we took responsibility for the human universe. Reality is not happening to us; it is a creative process that we materialize out of the invisible stuff of consciousness.

The demon of duality has done much mischief. We are entangled in the divisiveness described by Ralph Waldo Emerson:

Things are in the saddle,

And ride mankind.

There are two laws discrete,

Not reconciled-.

Law for man, and law for thing ...

I have overstepped my brief, to describe a specific interpretation of quantum mechanics. But nothing mechanical will explain reality or ourselves or how to get out of our many predicaments. Consciousness answers all of these riddles, and even if we cannot see where the trail leads, we certainly know where it begins.

Open Access This article is distributed under the terms of the Creative Commons Attribution 4.0 International License (http:// creativecommons.org/licenses/by/4.0/), which permits unrestricted use, distribution, and reproduction in any medium, provided you give appropriate credit to the original author(s) and the source, provide a link to the Creative Commons license, and indicate if changes were made.

Deepak Chopra MD, FACP, founder of The Chopra Foundation and cofounder of The Chopra Center for Wellbeing, is a world-renowned pioneer in integrative medicine and personal transformation, and is Board Certified in Internal Medicine, Endocrinology and Metabolism. He is a Fellow of the American College of Physicians and a member of the American Association of Clinical Endocrinologists. Chopra is the author of more than 85 books translated into over 43 languages, including numerous New York Times bestsellers.www.deepakchopra.com

Publisher's Note Springer Nature remains neutral with regard to jurisdictional claims in published maps and institutional affiliations. 